Commun. Korean Math. Soc. 28 (2013), No. 3, pp. 571-580

http://dx.doi.org/10.4134/CKMS.2013.28.3.571

\title{
A NOTE ON THE LORENTZIAN LIMIT CURVE THEOREM
}

\author{
JONG-GUG YUN
}

Abstract. In this paper, we extend the familiar limit curve theorem in [2] to a situation where each causal curve lies in a sequence of compact interpolating spacetimes converging to a limit Lorentz space in the sense of Lorentzian Gromov-Hausdorff distance.

\section{Introduction}

The familiar limit curve theorem in [2] guarantees the existence of limit causal curves for a sequence $\left\{\gamma_{n}\right\}$ of causal curves having points of accumulation in a given spacetime.

In general, the compactness of the space of causal curves between two points of a spacetime plays an essential role in the study of global structure of the spacetime. In fact, many of the singularity theorems in general relativity and a recent proof of positive energy theorem as well as Lorentzian splitting theorem depend on the compactness of the space of causal curves in the spacetime ([6]).

In 1996, R. Sorkin and E. Woolgar [7] introduced the concept of $K$-causal relation and extended the standard compactness theorem of causal curves to a situation where the differentiability of metric of the spacetime is only $C^{0}$. From the viewpoint of modern physics, there are strong evidences that our spacetime is not smooth but just a structure which looks like a smooth manifold on a scale larger than the Plank length. So lower degree of smoothness of spacetimes is of importance in the study of modern model of spacetime including theories of quantum gravity.

Another approach to spacetimes with lower degree of smoothness was introduced by J. Noldus using Lorentzian Gromov-Hausdorff theory ([1], [3], [4], $[5])$.

In [3], a Lorentzian notion of Gromov-Hausdorff distance $d_{G H}(G H)$ and the generalized Gromov-Hausdorff uniformity $(G G H)$ were first defined and some properties of limit space $M$ of GGH-Cauchy sequences of compact, interpolating spacetimes $\left\{M_{\alpha}\right\}$ were shown and proved.

Received August 8, 2012.

2010 Mathematics Subject Classification. Primary 53C20; Secondary 57S20.

Key words and phrases. limit curve theorem, Lorentzian Gromov-Hausdorff distance.

(C)2013 The Korean Mathematical Society 
In general, the limit space $M$ needs not to be a smooth manifold, but belongs to a class of Lorentz spaces. Here, in connection with the compactness of causal curves, a natural question which arises is whether we could guarantee the existence of limit causal curve $\gamma$ in the limit space $M$ of a sequence of causal curves $\left\{\gamma_{\alpha}\right\}$, where each $\gamma_{\alpha}$ lies in $M_{\alpha}$ rather than a single spacetime $M$ and $M_{\alpha}$ converges to $\mathrm{M}$ in the sense of Lorentzian Gromov-Hausdorff distance.

The main purpose of this paper is to answer this question in an affirmative way and investigate the type of the convergence of $\gamma_{\alpha}$ to $\gamma$.

In the next section, we introduce some definitions and notions which are necessary to state our main result.

\section{Preliminaries and main results}

We begin with the notion of Gromov-Hausdorff distance $d_{G H}(G H)$ between two globally hyperbolic compact interpolating (i.e., with spacelike future and past boundaries) spacetimes.

Definition 2.1. We call $(M, g)$ and $(N, h) \epsilon$-close if and only if there exist mappings $\psi: M \rightarrow N, \xi: N \rightarrow M$, not necessarily continuous, such that

$$
\begin{aligned}
& \left|d_{h}\left(\psi\left(p_{1}\right), \psi\left(p_{2}\right)\right)-d_{g}\left(p_{1}, p_{2}\right)\right| \leq \epsilon \forall p_{1}, p_{2} \in M, \\
& \left|d_{g}\left(\xi\left(q_{1}\right), \xi\left(q_{2}\right)\right)-d_{h}\left(q_{1}, q_{2}\right)\right| \leq \epsilon \forall q_{1}, q_{2} \in N,
\end{aligned}
$$

where $d_{h}$ and $d_{g}$ are the Lorentz distance defined from $g$ and $h$, respectively.

The Gromov-Hausdorff distance $d_{G H}((M, g),(N, h))$ is defined as the infimum over all $\epsilon$ such that $(M, g)$ and $(N, h)$ are $\epsilon$-close.

In [1], the author constructed a very useful metric $D_{M}$ for any compact interpolating spacetime $(M, g)$ such that every $d_{g}$ isometry is a $D_{M}$ isometry.

Definition 2.2. Let $(M, g)$ be a compact interpolating spacetime. The strong metric $D_{M}$ is defined as

$$
D_{M}(p, q)=\max _{r \in M}\left|d_{g}(p, r)+d_{g}(r, p)-d_{g}(q, r)-d_{g}(r, q)\right| .
$$

It was shown in [3] that the manifold, strong, and Alexandrov topology coincide for any compact interpolating spacetime.

We also introduce the following definition of Generalized Lorentzian GromovHausdorff uniformity (GGH):

Definition 2.3. We call $(M, g)$ and $(N, h)(\epsilon-\delta)$ close if and only if there exist mappings $\psi: M \rightarrow N, \xi: N \rightarrow M$ such that

$$
\begin{aligned}
& \left|d_{h}\left(\psi\left(p_{1}\right), \psi\left(p_{2}\right)\right)-d_{g}\left(p_{1}, p_{2}\right)\right| \leq \epsilon \forall p_{1}, p_{2} \in M, \\
& \left|d_{g}\left(\xi\left(q_{1}\right), \xi\left(q_{2}\right)\right)-d_{h}\left(q_{1}, q_{2}\right)\right| \leq \epsilon \forall q_{1}, q_{2} \in N
\end{aligned}
$$

and $D_{M}(p, \xi \circ \psi(p)) \leq \delta, D_{N}(q, \psi \circ \xi(q)) \leq \delta$ for all $p \in M$ and $q \in N$. 
As stated before, the limit space $M$ of GH (or GGH)-Cauchy sequence of compact interpolating spacetime need not to be a smooth manifold, but it belongs to a class of Lorentz space which can be defined as follows:

Definition 2.4. Let $M$ be a set. Lorentz distance is a function $d: M \times M \rightarrow$ $\mathbb{R}^{+} \cup\{\infty\}$ which satisfies for all $x, y, z \in M$ :

- $d(x, x)=0$,

- $d(x, y)>0$ implies $d(y, x)=0$ (antisymmetry),

- if $d(x, y) d(y, z)>0$, then $d(x, z) \leq d(x, y)+d(y, z)$ (reverse triangle inequality).

Definition 2.5. A Lorentz space is a pair $(M, d)$, where $M$ is a set and $d$ is a Lorentz distance on $M$ such that $\left(M, D_{M}\right)$ is a compact metric space with $D_{M}$ the strong metric induced by $d$.

We also note that any limit Lorentz space $(M, d)$ of a GH-Cauchy sequence $\left(M_{i}, d_{i}\right)$ of Lorentz spaces can be regarded as the limit space of GGH-Cauchy sequence $\left(M_{i}, d_{i}\right)$ as well (For details, see Theorem 26 in [4]).

In general, it is not easy problem how to define a natural causal relation on the limit Lorentz space and it is difficult especially on the degenerate regions of the limit space, which does not contain any set of the form $I^{+}(p) \cap I^{-}(q)$ for $p \ll q$. Here, we used the standard notation $\ll$ for the chronological relation between $p$ and $q$ and $I^{+}(p)=\{r \in M \mid p \ll r\}$ for the chronological future of $p$ and $I^{-}(p)=\{r \in M \mid r \ll p\}$ for the chronological past of $p$. In fact, as was pointed in [4], it is not entirely clear whether defining a causal relation on such regions is physically meaningful or not, and what degenerate region would mean from the physical point of view.

To avoid these difficulties, a controlling mechanism was suggested in [4] to prohibit the limit space from containing degenerate regions as follows.

Let $\alpha: \mathbb{R}^{+} \rightarrow \mathbb{R}^{+}$be a strictly increasing, continuous function such that $\alpha(x) \leq x$ for all $x \in \mathbb{R}^{+}$. We say that $(M, g)$ has the $C_{\alpha}^{+}$or $C_{\alpha}^{-}$property if and only if for any $\epsilon$ with $0<\epsilon \leq \operatorname{tdiam}(M)\left(:=\max _{p, q \in M} d(p, q)\right)$ we have that, respectively:

- $\alpha(\epsilon) \leq \min _{p \in M \downarrow \epsilon}\left[\max _{r \in \overline{B_{D_{M}}(p, \epsilon)}} d_{g}(p, r)\right] \leq \epsilon$,

- $\alpha(\epsilon) \leq \min _{p \in M^{\uparrow \epsilon}}\left[\max _{r \in \overline{B_{D_{M}}(p, \epsilon)}} d_{g}(r, p)\right] \leq \epsilon$, where $M^{\downarrow \epsilon}=\{p \in M \mid p \notin$ $\left.\left(\partial_{F} M\right)^{\epsilon}\right\}$ and $M^{\uparrow \epsilon}=\left\{p \in M \mid p \notin\left(\partial_{P} M\right)^{\epsilon}\right\}$.

Here, we denoted the future (resp. past) boundary of $M$ by $\partial_{F} M$ (resp. $\left.\partial_{P} M\right)$ and used the notation $A^{\epsilon}=\left\{q \in M \mid \exists a \in A\right.$ such that $\left.D_{M}(a, q)<\epsilon\right\}$ for any $A \subset M$.

It was shown in [4] that the GGH-limit space $(M, d)$ of a sequence $\left\{\left(M_{i}, g_{i}\right)\right\}$ satisfying the $C_{\alpha}^{+}$property has the $C_{\alpha}^{+}$property too (and likewise for $C_{\alpha}^{-}$). That is, $C_{\alpha}^{+}, C_{\alpha}^{-}$properties are stable under GGH-convergence (See Theorem 21 in [4]).

Furthermore, if the limit Lorentz space $(M, d)$ satisfies the $C_{\alpha}^{+}, C_{\alpha}^{-}$property, then a suitable causal structure on $(M, d)$, which was first defined by R. Sorkin 
and E. Woolgar can be introduced. We call it $K$-causal relation on $M$ and introduce the definition of it here.

Recall first that the chronological relation $\ll$ on $M$ can be defined by

$$
p \ll q \text { if and only if } d(p, q)>0 \text {. }
$$

Then $K$-causal relation $\prec$ on $M$ is defined using the topology of $M$ as follows.

Definition 2.6. We say $p \prec q$ if and only if $q \in K^{+}(p)$, where $K^{+}(p)$ is the smallest, topologically closed, partial order in $M \times M$ containing $I^{+}(p)\left(K^{-}(p)\right.$ can be defined dually).

Remark 2.1. We note that the requirement of "partial order" in Definition 2.6 contains additional conditions (i.e., reflexive and antisymmetric) when compared with the original definition of $K^{+}$in [7] where only transitivity is required).

Now we introduce the concept of causal curves in a Lorentz space $M$ with a causal relation $\prec$ as in [4].

Definition 2.7. Let $(M, d)$ be a Lorentz space. Assume $a<b$ and let $\gamma$ : $[a, b] \rightarrow M$ be a continuous (with respect to the strong topology) mapping such that for all $a \leq t<s \leq b: \gamma(t) \prec \gamma(s)(\gamma(t) \ll \gamma(s))$; then $\gamma$ is called a basic, causal (timelike) curve.

For any topological space $X$, the basic open sets $\mathcal{B}\left(A_{0} ; A_{1} ; \cdots ; A_{n}\right)\left(A_{i}\right.$ 's are open in $\mathrm{X}$ ) of the Vietoris topology on the space $2^{X}$ of nonempty closed subsets can be defined as follows:

$$
C \in \mathcal{B}\left(A_{0} ; A_{1} ; \cdots ; A_{n}\right) \Longleftrightarrow C \subseteq A_{0} \text { and } C \text { meets } A_{i} \text { for } i=1, \ldots, n \text {. }
$$

It is known in [7] that $2^{X}$ with the Vietoris topology is compact if $X$ is compact. It is also shown that the Viertoris limit of a sequence of $K$-causal curves is a $K$-causal curve using only the topological arguments.

For any sequence of compact, interpolating spacetime $\left\{\left(M_{\alpha}, d_{\alpha}\right)\right\}$ converging to a Lorentz space $(M, d)$ in the sense of GH-distance, we can extend each strong metric $D_{\alpha}$ on $M_{\alpha}$ and $D_{M}$ on $M$ to an admissible metric $\widetilde{D}$ on $\widetilde{M}=$ $M \coprod\left(\coprod_{\alpha} M_{\alpha}\right)$ as in Theorem 17 of [4].

Keeping this extension of metric in mind, it makes sense to say that a sequence of causal curves $\left\{\gamma_{\alpha}\right\}$, where $\gamma_{\alpha} \subset M_{\alpha}$, converges to a causal curve $\Gamma$ in $M$ in the Vietoris topology on $2^{(\widetilde{M}, \widetilde{D})}$.

Now we are in a position to state our main result as follows.

Theorem 2.1. Let $\left\{M_{\alpha}\right\}$ be a sequence of compact, interpolating spacetimes satisfy $C_{\alpha}^{ \pm}$properties and $\gamma_{\alpha}:[a, b] \rightarrow M_{\alpha}$ be a causal curve in $M_{\alpha}$. Assume that $\left\{M_{\alpha}\right\}$ converges to a Lorentz space $M$ with $K$-causal relation in the sense of $G H$-distance. Then there exists a subsequence $\left\{\gamma_{\beta}\right\}$ of $\left\{\gamma_{\alpha}\right\}$ such that $\left\{\gamma_{\beta}\right\}$ converges to a causal curve $\Gamma$ in $M$ in the Vietoris topology on $2^{(\widetilde{M}, \widetilde{D})}$, where $(\widetilde{M}, \widetilde{D})$ is defined as above. 
In Section 4, we shall also investigate the convergence of the lengths of the causal curves $\gamma_{\alpha}$ and obtain a result as we expected.

Theorem 2.2. Let $\left\{\gamma_{\beta}\right\}$ and $\Gamma$ be as in Theorem 2.1. Then we have

$$
\limsup _{\beta \rightarrow \infty} L\left[\gamma_{\beta}\right] \leq L[\Gamma] \text {. }
$$

\section{Proof of Theorem 2.1}

We first note that for each $\alpha$, we have a map $\psi_{\alpha}: M_{\alpha} \rightarrow M$ which makes $M_{\alpha}$ and $M\left(\epsilon_{\alpha}, \epsilon_{\alpha}\right)$-close $\left(\epsilon_{\alpha} \rightarrow 0\right.$ as $\left.\alpha \rightarrow \infty\right)$, since $M$ can be regard as the GGH-limit space of $\left\{M_{\alpha}\right\}$.

Let $\Gamma_{\alpha}=\psi_{\alpha} \circ \gamma_{\alpha}$ and consider the closure $\bar{\Gamma}_{\alpha}$ of $\Gamma_{\alpha}$ in $M$ for each $\alpha$. Since $\left\{\bar{\Gamma}_{\alpha}\right\}$ is a sequence of closed subsets of $M$ which is compact, we have a closed subset $\Gamma$ in $M$ such that a subsequence $\left\{\bar{\Gamma}_{\beta}\right\}$ of $\left\{\bar{\Gamma}_{\alpha}\right\}$ converges to $\Gamma$ in the Vietoris topology in $M$.

Now we claim that $\Gamma$ is a basic, causal curve in $M$ by showing that it satisfies the hypotheses of the following theorem.

Theorem 3.1 ([7]). Let $\Gamma$ be a set provided with both a linear order and a topology such that

- with respect to the topology it is compact and connected and contains a countable dense subset,

- with respect to the order it has both a minimal and a maximal element, and

- (with respect to both) it has the property that $\ll x, y \gg=\{r \in M \mid x \prec$ $r \prec y\}$ is topologically closed for all $x, y \in \Gamma$.

Then $\Gamma$ is isomorphic to the interval $[0,1] \subset \mathbb{R}$ by a simultaneous order and topological isomorphism.

Note that we can apply the same arguments as in Theorem 20 in [7] to verify that $\Gamma$ has a countable dense subset, minimal and maximal elements and every interval in $\Gamma$ is topologically closed. Since $\Gamma$ is compact clearly, we need only to show that it is connected and linearly ordered to verify that $\Gamma$ satisfies the hypotheses in Theorem 3.1 .

We first show that $\Gamma$ is connected as follows. Note that the number of components of $\Gamma$ should be finite by the compactness of $\Gamma$, so we may let $\left\{\Gamma_{1}, \Gamma_{2}, \ldots, \Gamma_{n}\right\}$ be the finite components of $\Gamma$.

For simplicity, we assume $n=2$ (we can argue similarly for the general case) and we first claim $\Gamma_{1}$ is closed in $M$. This can easily be seen by noting that every accumulation point of $\Gamma_{1}$ should be in $\Gamma=\Gamma_{1} \coprod \Gamma_{2}$ since $\Gamma$ is closed and it cannot be in $\Gamma_{2}$. Consequently, every accumulation point of $\Gamma_{1}$ should be in $\Gamma_{1}$ itself and this says that $\Gamma_{1}$ is closed. $\Gamma_{2}$ is closed by the same arguments. Now we can choose $x_{1} \in \Gamma_{1}$ and $x_{2} \in \Gamma_{2}$ such that $D_{M}\left(x_{1}, x_{2}\right)=\min \left\{D_{M}(x, y) \mid x \in \Gamma_{1}, y \in \Gamma_{2}\right\}>0$ which is possible, since $\Gamma_{1}, \Gamma_{2}$ are compact and disjoint. 
Letting $\epsilon=D_{M}\left(x_{1}, x_{2}\right)>0$, we know that $V_{1}:=N\left(\Gamma_{1}, \frac{\epsilon}{3}\right)=\{y \in$ $\left.M \mid D_{M}\left(\Gamma_{1}, y\right)<\frac{\epsilon}{3}\right\}$ and $V_{2}:=N\left(\Gamma_{2}, \frac{\epsilon}{3}\right)=\left\{y \in M \mid D_{M}\left(\Gamma_{2}, y\right)<\frac{\epsilon}{3}\right\}$ are disjoint open sets containing $\Gamma_{1}$ and $\Gamma_{2}$ respectively and satisfy the following:

$$
D_{M}(x, y)>\frac{\epsilon}{3} \text { for all } x \in V_{1}, y \in V_{2} \text {. }
$$

Since $\left\{\bar{\Gamma}_{\beta}\right\}$ converges to $\Gamma$ in the Vietoris topology, we may assume that $\left\{\bar{\Gamma}_{\beta}\right\} \subset V_{1} \coprod V_{2}$ for all $\beta$ (by taking a subsequence if necessary).

Now we let $U_{1}=\left\{\left.x \in \gamma_{\beta}\right|_{[a, b]} \mid \psi_{\beta}(x) \in V_{1}\right\}, U_{2}=\left\{\left.x \in \gamma_{\beta}\right|_{[a, b]} \mid \psi_{\beta}(x) \in V_{2}\right\}$ and claim that $\inf \left\{D_{\alpha}(x, y) \mid x \in U_{1}, y \in U_{2}\right\}=0$.

Suppose that $\tau=\inf \left\{D_{\alpha}(x, y) \mid x \in U_{1}, y \in U_{2}\right\}>0$ and consider $\mathcal{U}$, a family of open balls with centers on $\left.\gamma_{\beta}\right|_{[a, b]}$ and radius $\frac{\tau}{3}$. That is, $\mathcal{U}=$ $\left\{B_{\beta}\left(z, \frac{\tau}{3}\right)\left|z \in \gamma_{\beta}\right|_{[a, b]}\right\}$.

By the compactness of $\left.\gamma_{\beta}\right|_{[a, b]}$, we have a family of finite open balls $\left\{B_{\beta}\left(z_{1}, \frac{\tau}{3}\right)\right.$, $\left.\ldots, B_{\beta}\left(z_{n}, \frac{\tau}{3}\right)\right\}$ and each $B_{\beta}\left(z_{i}, \frac{\tau}{3}\right)$ contains an element in $U_{1}$ or $U_{2}$ exclusively. But this gives a disconnection of $\left.\gamma_{\beta}\right|_{[a, b]}$ which is a contradiction.

We now note from the proof of Theorem 16 in [4] that

$$
\left|D_{M}\left(\psi_{\beta}(x), \psi_{\beta}(y)\right)-D_{\beta}(x, y)\right|<4 \epsilon_{\beta} \forall x,\left.y \in \gamma_{\beta}\right|_{[a, b]} .
$$

So arbitrary closeness between $\left.x_{1} \in U_{1} \subset \gamma_{\beta}\right|_{[a, b]}$ and $\left.x_{2} \in U_{2} \subset \gamma_{\beta}\right|_{[a, b]}$ implies arbitrary closeness between $\psi_{\beta}\left(x_{1}\right) \in V_{1}$ and $\psi_{\beta}\left(x_{2}\right) \in V_{2}$ in $M$.

But this contradicts to (3.1) and we conclude that $\Gamma$ is connected.

Next, we show that $\Gamma$ is a linearly ordered set. For this purpose, we need to show that for any $p, q \in \Gamma$, they are related by $\prec$.

In case that $p$ or $q$ is the minimal or maximal element, we have nothing to prove and assume that the two points are neither minimal nor maximal. Then we can say that neither point is in the future or past boundary of $M$. For, if $q$ is in the future boundary $\partial_{F} M$, then we have $I^{+}(q)=\emptyset$ and $K^{+}(q)=\{q\}$ by the definition of $K$-causal relation, which implies that $q$ is the maximal element. The same argument holds for the past boundary of $M$.

Now since $\left\{\bar{\Gamma}_{\beta}\right\}$ converges to $\Gamma$ in the Vietoris topology, we may consider sequences $\left\{\psi_{\beta}\left(p_{\beta}\right)\right\}$ and $\left\{\psi_{\beta}\left(q_{\beta}\right)\right\}$, where $p_{\beta}, q_{\beta} \in \gamma_{\beta}$ and $\psi_{\beta}\left(p_{\beta}\right) \rightarrow p, \psi_{\beta}\left(q_{\beta}\right) \rightarrow$ $q$ in $\left(M, D_{M}\right)$ (passing to a subsequence if necessary). We may also assume that $q_{\beta}$ is in the causal future of $p_{\beta}$ without loss of generality.

From now on, we claim that there is a sequence $\left\{r_{\beta_{k}}\right\}$, where $\left\{\beta_{k}\right\} \subset\{\beta\}$ such that $\left\{\psi_{\beta_{k}}\left(r_{\beta_{k}}\right)\right\} \rightarrow q$ in $\left(M, D_{M}\right)$ and $\psi_{\beta_{k}}\left(p_{\beta_{k}}\right) \ll \psi_{\beta_{k}}\left(r_{\beta_{k}}\right)$ for each $\beta_{k}$.

Note first that for sufficiently large $N_{1} \in \mathbb{N}$, we have $B_{M}\left(q, \frac{1}{N_{1}}\right) \cap \partial_{F} M=\phi$, since $q \notin \partial_{F} M$. Then by the $C_{\alpha}^{+}$property, there exists an $r^{\prime} \in \overline{B_{M}\left(q, \frac{1}{2 N_{1}}\right)}$ such that $d\left(q, r^{\prime}\right)=\alpha\left(\frac{1}{2 N_{1}}\right)>0$. Let $\left\{r_{\beta}\right\}$ be a sequence in $(\widetilde{M}, \widetilde{D})$ such that $r_{\beta} \in M_{\beta}(\forall \beta)$ and $\left\{r_{\beta}\right\} \rightarrow r^{\prime}$ in $(\widetilde{M}, \widetilde{D})$. Then there exists a $\beta^{1,1}>0$ such that $\widetilde{D}\left(r_{\beta}, r^{\prime}\right)<\frac{1}{N_{1}}$ for all $\beta$ with $\beta \geq \beta^{1,1}$. 
On the other hand, since $d_{\beta}\left(q_{\beta}, r_{\beta}\right)$ converges to $d\left(q, r^{\prime}\right)=\alpha\left(\frac{1}{2 N_{1}}\right)>0$ as $\beta \rightarrow \infty$ (Refer to the proof of Theorem 17 in [4] for details), we know that there exists a $\beta^{1,2}$ such that $q_{\beta} \ll r_{\beta}$ for all $\beta$ with $\beta \geq \beta^{1,2}$.

Thus, by the reverse triangle inequality, we have

$$
d_{\beta}\left(p_{\beta}, r_{\beta}\right) \geq d_{\beta}\left(q_{\beta}, r_{\beta}\right) \sim \alpha\left(\frac{1}{2 N_{1}}\right) \text { for all } \beta \text { with } \beta \geq \beta^{1,2} .
$$

Now we obtain

$$
\begin{aligned}
d\left(\psi_{\beta}\left(p_{\beta}\right), \psi_{\beta}\left(r_{\beta}\right)\right) & \geq d\left(p_{\beta}, r_{\beta}\right)-\epsilon_{\beta} \\
& \geq d_{\beta}\left(q_{\beta}, r_{\beta}\right)-\epsilon_{\beta} \\
& \sim \alpha\left(\frac{1}{2 N_{1}}\right)-\epsilon_{\beta}>0 \text { for all sufficiently large } \beta .
\end{aligned}
$$

Consequently, there exists a $\beta^{1,3}>0$ such that $\psi_{\beta}\left(p_{\beta}\right) \ll \psi_{\beta}\left(r_{\beta}\right)$ for all $\beta$ with $\beta \geq \beta^{1,3}$. Now we let $\beta_{1}=\max \left\{\beta^{1,1}, \beta^{1,2}, \beta^{1,3}\right\}$ and note that $\widetilde{D}\left(r_{\beta_{1}}, r^{\prime}\right)<$ $\frac{1}{N_{1}}, r^{\prime} \in \overline{B_{M}\left(q, \frac{1}{2 N_{1}}\right)}$ and $\psi_{\beta_{1}}\left(p_{\beta_{1}}\right) \ll \psi_{\beta_{1}}\left(r_{\beta_{1}}\right)$.

Let $N_{k}=N_{1}+(k-1)(k=2,3, \ldots)$ and proceed the same process to obtain $r^{k} \in \overline{B_{M}\left(q, \frac{1}{2 N_{k}}\right)}$ and $\beta_{k}$ such that $\psi_{\beta_{k}}\left(p_{\beta_{k}}\right) \ll \psi_{\beta_{k}}\left(r_{\beta_{k}}\right)(k=2,3, \ldots)$ and $\widetilde{D}\left(r^{k}, r_{\beta_{k}}\right)<\frac{1}{N_{k}}=\frac{1}{N_{1}+(k-1)}$.

We now have

$$
\widetilde{D}\left(q, \psi_{\beta_{k}}\left(r_{\beta_{k}}\right)\right) \leq \widetilde{D}\left(q, r^{k}\right)+\widetilde{D}\left(r^{k}, r_{\beta_{k}}\right)+\widetilde{D}\left(r_{\beta_{k}}, \psi_{\beta_{k}}\left(r_{\beta_{k}}\right)\right),
$$

where the three terms of the right hand side of the above inequality converge to zero as $k \rightarrow \infty$ by the construction of $r_{k}, r_{\beta_{k}}$ and the inequality $\widetilde{D}\left(x_{\alpha}, \psi_{\alpha}\left(x_{\alpha}\right)\right)<\epsilon_{\alpha}+\frac{3 \epsilon_{\alpha}}{2}=\frac{5 \epsilon_{\alpha}}{2}$ for all $x_{\alpha} \in M_{\alpha}$ (See p. 70 in [4] for reference).

Thus we obtain that $\psi_{\beta}\left(r_{\beta_{k}}\right)$ converges to $q$ in $\left(M, D_{M}\right)$. Now $p \prec q$ follows from the closeness of $K^{+}$, which proves that $\Gamma$ is a linearly ordered set.

Now we claim that $\left\{\gamma_{\beta}\right\}$ converges to $\Gamma$ in the Vietoris topology on $(\widetilde{M}, \widetilde{D})$. Recall that our claim is equivalent to show that $\left\{\gamma_{\beta}\right\}$ converges to $\Gamma$ in the both "upper" and "lower" topology on $(\widetilde{M}, \widetilde{D})([7])$.

In the lower topology, $\left\{\gamma_{\beta}\right\}$ converges to $\Gamma$ if and only if the $\left\{\gamma_{\beta}\right\}$ eventually "meet" every open set $U \subseteq \widetilde{M}$ which meets $\Gamma$. In the upper topology (also called the $C^{0}$ topology), $\left\{\gamma_{\beta}\right\}$ converges to $\Gamma$ if and only if the $\left\{\gamma_{\beta}\right\}$ are eventually "included" in every open set $U \subseteq \widetilde{M}$ which includes $\Gamma$ itself (For details, refer to p. 32 in [7]).

We start with the convergence in the lower topology on $(\widetilde{M}, \widetilde{D})$.

Let $U \subseteq \widetilde{M}$ be any open set which meets $\Gamma$ at $p$ and consider an $\epsilon$-ball $B_{\widetilde{M}}(p, \epsilon)$ with $(p \in) B_{\widetilde{M}}(p, \epsilon) \subset U$. Since $\left\{\bar{\Gamma}_{\beta}\right\}$ converges to $\Gamma$ in the Vietoris topology on $\left(M, D_{M}\right)$, we can easily see that there exists a $\beta_{1}>0$ such that $B_{M}\left(p, \frac{\epsilon}{2}\right) \cap \Gamma_{\beta} \neq \emptyset$ for all $\beta$ with $\beta \geq \beta_{1}$, where we note $B_{M}\left(p, \frac{\epsilon}{2}\right)=B_{\widetilde{M}}\left(p, \frac{\epsilon}{2}\right) \cap$ $M$. 
We denote an element in $B_{M}\left(p, \frac{\epsilon}{2}\right) \cap \Gamma_{\beta}$ by $\psi_{\beta}\left(p_{\beta}\right)$, where $p_{\beta} \in \gamma_{\beta}$ for $\beta \geq \beta_{1}$

Since $\left\{M_{\beta}\right\}$ converges to $M$ in the GGH-sense, we also know that there exists a $\beta_{2}>0$ such that $\widetilde{D}\left(p_{\beta}, \psi_{\beta}\left(p_{\beta}\right)\right)<\frac{\epsilon}{2}$ for all $\beta \geq \beta_{2}$.

We now have $\widetilde{D}\left(p_{\beta}, p\right) \leq \widetilde{D}\left(p_{\beta}, \psi_{\beta}\left(p_{\beta}\right)\right)+\widetilde{D}\left(\psi_{\beta}\left(p_{\beta}\right), p\right)<\frac{\epsilon}{2}+\frac{\epsilon}{2}=\epsilon$ for all $\beta$ with $\beta \geq \max \left\{\beta_{1}, \beta_{2}\right\}$. This means that $B_{\widetilde{M}}(p, \epsilon) \cap \gamma_{\beta} \neq \emptyset$ for all $\beta$ with $\beta \geq \max \left\{\beta_{1}, \beta_{2}\right\}$, which implies that $U \cap \gamma_{\beta} \neq \emptyset$ for all $\beta$ with $\beta \geq \max \left\{\beta_{1}, \beta_{2}\right\}$ since $B_{\widetilde{M}}(p, \epsilon) \subset U$. Thus we conclude that $\left\{\gamma_{\beta}\right\}$ converges to $\Gamma$ in the lower topology on $(\widetilde{M}, \widetilde{D})$ (In the terminology in [2], $\Gamma$ is the limit curve of $\left.\left\{\gamma_{\alpha}\right\}\right)$.

We next show that $\left\{\gamma_{\beta}\right\}$ converges to $\Gamma$ in the upper topology $\left(C^{0}\right.$-topology) on $(\widetilde{M}, \widetilde{D})$. Let $U \subseteq \widetilde{M}$ be any open set which contains $\Gamma$ and for each point $p \in \Gamma$ consider open balls $B_{\widetilde{M}}(p, \delta(p)), B_{\widetilde{M}}\left(p, \frac{\delta(p)}{2}\right)$ for some $\delta(p)>0$ such that $(p \in) B_{\widetilde{M}}\left(p, \frac{\delta(p)}{2}\right) \subset B_{\widetilde{M}}(p, \delta(p)) \subset U$.

Since $\left\{B_{M}\left(p, \frac{\delta(p)}{2}\right) \mid p \in \Gamma\right\}$ is an open covering of $\Gamma$ in $M$ and $\Gamma$ is compact, we have finite $p_{1}, \ldots, p_{n}$ on $\Gamma$ such that $\Gamma \subset \cup_{i=1}^{n} B_{M}\left(p_{i}, \frac{\delta(p)}{2}\right) \subset U$.

By the convergence of $\left\{\bar{\Gamma}_{\beta}\right\}$ to $\Gamma$ in the Vietoris topology on $\left(M, D_{M}\right)$ we know that there exists a $\beta_{3}>0$ such that $\bar{\Gamma}_{\beta} \subset \cup_{i=1}^{n} B_{M}\left(p_{i}, \frac{\delta(p)}{2}\right)$ for all $\beta$ with $\beta \geq \beta_{3}$.

Let $\delta_{0}=\frac{1}{2} \min \left\{\delta\left(p_{1}\right), \ldots, \delta\left(p_{n}\right)\right\}$ and note that there exists a $\beta_{4}>0$ such that $\widetilde{D}\left(p_{\beta}, \psi_{\beta}\left(p_{\beta}\right)\right)<\delta_{0}$ for all $p_{\beta} \in \gamma_{\beta}$ and $\beta \geq \beta_{4}$.

Now, for any $p_{\beta} \in \gamma_{\beta}$ where $\beta \geq \max \left\{\beta_{3}, \beta_{4}\right\}$, take $p_{i}(1 \leq i \leq n)$ such that $\psi_{\beta}\left(p_{\beta}\right) \in B_{M}\left(p_{i}, \frac{\delta\left(p_{i}\right)}{2}\right)$, which is possible since $\psi_{\beta}\left(p_{\beta}\right) \in \Gamma_{\beta} \subset \bar{\Gamma}_{\beta} \subset$ $\cup_{i=1}^{n} B_{M}\left(p_{i}, \frac{\delta\left(p_{i}\right)}{2}\right)$ for all $\beta \geq \beta_{3}$.

We then have the following inequalities, for all $\beta \geq \max \left\{\beta_{3}, \beta_{4}\right\}$,

$$
\begin{aligned}
\widetilde{D}\left(p_{\beta}, p_{i}\right) & \leq \widetilde{D}\left(p_{\beta}, \psi_{\beta}\left(p_{\beta}\right)\right)+\widetilde{D}\left(\psi_{\beta}\left(p_{\beta}\right), p_{i}\right) \\
& \leq \delta_{0}+\frac{\delta\left(p_{i}\right)}{2} \leq \delta\left(p_{i}\right) .
\end{aligned}
$$

Thus if $\beta \geq \max \left\{\beta_{3}, \beta_{4}\right\}$, then every $p_{\beta}$ on $\gamma_{\beta}$ is contained in some ball $B_{\widetilde{M}}\left(p_{i}, \delta\left(p_{i}\right)\right)$ in $(\widetilde{M}, \widetilde{D})$. This means that $\gamma_{\beta} \subset \cup_{i=1}^{n} B_{\widetilde{M}}\left(p_{i}, \delta\left(p_{i}\right)\right) \subset U$ for all $\beta \geq \max \left\{\beta_{3}, \beta_{4}\right\}$ in $(\widetilde{M}, \widetilde{D})$. So we conclude that $\left\{\gamma_{\beta}\right\}$ converges to $\Gamma$ in the upper topology. Consequently, $\left\{\gamma_{\beta}\right\}$ converges to $\Gamma$ in the Vietoris topology on $(\widetilde{M}, \widetilde{D})$ and we complete the proof of Theorem 2.1 .

\section{Proof of Theorem 2.2}

The main point of the proof of Theorem 2.2 is the following Lemma 4.1, which can be easily shown by the similar arguments in the proof of Lemma 22 in $[7]$.

Lemma 4.1. $\left\{\gamma_{\beta}(a)\right\}$ (resp. $\left.\left\{\gamma_{\beta}(b)\right\}\right)$ converges to the initial (resp. final) point of $\Gamma$ in $(\widetilde{M}, \widetilde{D})$. 
Proof. Since we know $\widetilde{D}\left(\gamma_{\beta}(a), \psi_{\beta}\left(\gamma_{\beta}(a)\right)\right) \rightarrow 0$ as $\beta \rightarrow \infty$ from p. 70 in [4], it suffices to show that $\psi_{\beta}\left(\gamma_{\beta}(a)\right)$ converges to the initial point of $\Gamma$ in $\left(M, D_{M}\right)$ (The same is true for the final point).

Note that we may assume without loss of generality that $\left\{\psi_{\beta}\left(\gamma_{\beta}(a)\right)\right\}$ converges to a point $\Gamma_{0}$ in $M$, since $\left(M, D_{M}\right)$ is compact.

We now claim that $\Gamma_{0}$ is the initial point of $\Gamma$. Because $\Gamma$ is the Vietorislimit of $\left\{\overline{\psi_{\beta}\left(\gamma_{\beta}\right)}\right\}$ and $\Gamma$ is a closed set, it is easy to check that $\Gamma_{0}$ should be contained in $\Gamma$ itself.

For any $p$ in $\Gamma$ and $\left\{\psi_{\beta}\left(q_{\beta}\right)\right\}$ converging to $q$ in $\left(M, D_{M}\right)$, we know that $q_{\beta}$ is in the causal future of $\gamma_{\beta}(a)$ since $\gamma_{\beta}$ is a causal curve and $q_{\beta} \in \gamma_{\beta}$. Recall that $\Gamma$ is linearly ordered, so we have either $\Gamma_{0} \prec q$ or $q \prec \Gamma$. But we can apply the same arguments as in the proof of Theorem 2.1 to show that $\Gamma_{0} \prec q$ is true noting that $q_{\beta}$ is in the causal future of $p_{\beta}:=\gamma_{\beta}(a)$ and $\psi_{\beta}\left(p_{\beta}\right)$ converges to $\Gamma_{0}$ in $(\widetilde{M}, \widetilde{D})$ (In case that $q$ is in the future boundary of $M$ it is the final point of $\Gamma$ automatically as stated previously in the proof of Theorem 2.1, so there is nothing to prove).

Thus we conclude that $\Gamma_{0}$ is the initial point of $\Gamma$.

Now for any partition $\Delta$ of $[a, b], \Delta=\left\{t_{i} \mid a=t_{0}<t_{1}<\cdots<t_{n}=b\right\}$ and for each $\Gamma\left(t_{i}\right)(i=0,1, \ldots, n)$, consider a sequence $\left\{p_{\beta}^{i}\right\}$ in $\widetilde{M}$ such that $p_{\beta}^{i} \in \gamma_{\beta}$ and $p_{\beta}^{i} \rightarrow \Gamma\left(t_{i}\right)$ as $\beta \rightarrow \infty$. Note that we can put $p_{\beta}^{0}=\gamma_{\beta}(a), p_{\beta}^{n}=\gamma_{\beta}(b)$ by the above Lemma 4.1 .

Then we have

$$
\sum_{i=1}^{n} d\left(\Gamma\left(t_{i-1}\right), \Gamma\left(t_{i}\right)\right)=\lim _{\beta \rightarrow \infty} \sum_{i=1}^{n} d_{\beta}\left(p_{\beta}^{i-1}, p_{\beta}^{i}\right) \geq \limsup _{\beta \rightarrow \infty} L\left[\gamma_{\beta}\right] .
$$

Since $L[\Gamma]=\inf _{\Delta}\left\{\sum_{i=1}^{n} d\left(\Gamma\left(t_{i-1}, t_{i}\right)\right\}\right.$, we conclude that $L[\Gamma] \geq \lim \sup _{\beta \rightarrow \infty}$ $L\left[\gamma_{\beta}\right]$.

This completes the proof of Theorem 2.2.

\section{References}

[1] L. Bombelli and J. Noldus, The moduli space of isometry classes of globally hyperbolic spacetimes, Classical Quantum Gravity 21 (2004), no. 18, 4429-4453.

[2] S. W. Hawking and G. F. R. Ellis, The Large Scale Structure of Space-Time, Cambidge University Press, 1973.

[3] J. Noldus, A Lorentzian Gromov-Hausdorff notion of distance, Classical Quantum Gravity 21 (2004), no. 4, 839-850.

[4] Lorentzian Gromov Hausdorff theory as a tool for quantum gravity kinematics, PhD thesis, Gent University, 2004.

[5] - The limit space of a Cauchy sequence of globally hyperbolic spacetimes, Classical Quantum Gravity 21 (2004), no. 4, 851-874.

[6] R. Penrose, R. D. Sorkin, and E. Woolgar, A positive mass theorem based on the focusing and retardation of null geodesics, gr-qc/9301015.

[7] R. Sorkin and E. Woolgar, A causal order for spacetimes with $C^{0}$ Lorentzian metrics: proof of compactness of the space of causal curves, Classical Quantum Gravity 13 (1996), no. $7,1971-1993$. 
Department of Mathematics Education

Korea National University of Education

Chungbuk 363-791, Korea

E-mail address: jgyun69@knue.ac.kr 